\title{
Observation of a subharmonic gap singularity in interlayer tunneling characteristics of $\mathrm{Bi}_{2} \mathrm{Sr}_{2} \mathrm{CaCu}_{2} \mathrm{O}_{8+\delta}$
}

\author{
V. M. Krasnov* \\ Department of Physics, Stockholm University, AlbaNova University Center, SE-10691 Stockholm, Sweden
}

(Dated: May 29, 2022)

\begin{abstract}
A subharmonic structure in Josephson junctions appears due to Andreev reflections within the junction. Here we report on experimental observation of a subharmonic half-gap singularity in interlayer tunneling characteristics of a layered high temperature superconductor $\mathrm{Bi}_{2} \mathrm{Sr}_{2} \mathrm{CaCu}_{2} \mathrm{O}_{8+\delta}$. The singularity is most pronounced in optimally doped crystals and vanishes with decreasing doping. It indicates existence of non-vanishing electronic density of states and certain metallic properties in the intermediate $\mathrm{BiO}$ layers, which grows stronger with increasing doping. This provides an additional coherent interlayer transport channel and can explain a gradual transition from an incoherent quasi-two-dimensional $c$-axis transport in underdoped to a coherent metallic transport in overdoped cuprates. Furthermore, due to a very small sub-gap current, the singularity allows unambiguous extraction of the superconducting gap, without distortion by self-heating.
\end{abstract}

PACS numbers: $74.72 . \mathrm{Hs} 74.45 .+\mathrm{c} 74.50 .+\mathrm{r} 74.25 . \mathrm{Jb}$

The mechanism of interlayer (c-axis) transport in cuprate high temperature superconductors remains an actively debated subject. A qualitative difference between metallic in-plane and non-metallic out-of-plane resistivities [1] is a strong indication for predominantly incoherent nature of $c$-axis transport, which is achieved by interlayer hopping or tunneling [2-7]. The tunneling nature of $c$-axis transport leads to appearance of the intrinsic Josephson effect between $\mathrm{CuO}_{2}$ planes in layered $\mathrm{Bi}_{2} \mathrm{Sr}_{2} \mathrm{CaCu}_{2} \mathrm{O}_{8+\delta}$ (Bi-2212) cuprates at temperatures below $T_{c}$ 8. However, the electronic system in cuprates is not strictly two-dimensional. This has been demonstrated by observation of bonding-antibonding bilayer splitting of electronic bands 9 . Indications for coherent transport were obtained in strong magnetic fields 10. Since the two dimensional superconductivity is suppressed by fluctuations [11, presence or absence of the coherent metallic transport in the $c$-axis direction, i.e., in the third dimension, and the mechanism of interlayer coupling remain to be important issues for understanding high temperature superconductivity.

The intrinsic Josephson effect provides an accurate way of probing weak interlayer coupling in cuprates. Due to a $d$-wave symmetry of the order parameter, the product of the Josephson critical current $I_{c}$ and the normal resistance $R_{n}$ in intrinsic junctions should strongly depend on the coherence (momentum conservation) upon tunneling. The $I_{c} R_{n}$ is maximum $\sim \Delta / e$ for coherent, and zero for completely incoherent tunneling 12 . Here $\Delta$ is the maximum value of the superconducting energy gap. Analysis of $I_{c} R_{n}$ in intrinsic Josephson junctions indicated that in overdoped $\mathrm{Bi}-2212$ interlayer tunneling is predominantly coherent $I_{c} R_{n} \sim \Delta / e$ [13, 14]. However, $e I_{c} R_{n} / \Delta$ rapidly decreases upon opening of the pseudogap in the underdoped state [13, 14. This may either

*Electronic address: Vladimir.Krasnov@fysik.su.se indicate that interlayer tunneling becomes progressively more incoherent with decreasing doping [13], or that the Fermi surface is reconstructed by the pseudogap [14].

Intrinsic Josephson junctions are characterized by low dissipation [15]. This is often taken as evidence for Superconductor-Insulator-Superconductor (SIS) structure of $\mathrm{Bi}-2212$, in which $\mathrm{S}$ are superconducting $\mathrm{CuO}_{2}-$ $\mathrm{Ca}-\mathrm{CuO}_{2}$ bilayers and $\mathrm{I}$ is the insulating $\mathrm{SrO}-2 \mathrm{BiO}-\mathrm{SrO}$ layer. Yet, this does not preclude that some of the layers in the $\mathrm{SrO}-2 \mathrm{BiO}-\mathrm{SrO}$ stack are metallic, like in case of SINIS ( $\mathrm{N}$ - is a normal metal) or SIS'IS junctions [16 18, provided that transparency of the I interface is sufficiently low. The metallic behavior of the intermediate layer is manifested in appearance of subharmonic gap structure [19 21] due to Andreev reflection of quasiparticles into Cooper pairs [22] at the SIN interface.

In this work we report on observation of the subharmonic gap singularity in intrinsic tunneling characteristics of small Bi-2212 mesa structures. This indicates presence of a finite electronic density of states in $\mathrm{BiO}$ layers. The singularity is most pronounced in optimally doped crystals and decreases with decreasing doping. The subgap singularity allows evaluation of the gap value at negligible self-heating. We demonstrate that the energy gap can be confidently extracted for more than two orders of magnitude variation of the dissipation power. This provides an important self-consistency check for intrinsic tunneling spectroscopy. Finally, we discuss consequences of the metallic behavior of $\mathrm{BiO}$ layers, which provides an additional coherent transport channel and can explain a gradual transition from a two-dimensional incoherent to a three-dimensional coherent $c$-axis transport with increasing doping.

We present data for three batches of single crystals: the Y-substituted $\mathrm{Bi}_{2} \mathrm{Sr}_{2} \mathrm{Ca}_{1-x} \mathrm{Y}_{x} \mathrm{Cu}_{2} \mathrm{O}_{8+\delta}, \mathrm{Bi}(\mathrm{Y})-$ 2212 ), with the maximum $T_{c} \simeq 94.5 \mathrm{~K}$; the lead-doped $\mathrm{Bi}_{2}-x \mathrm{~Pb}_{x} \mathrm{Sr}_{2} \mathrm{Ca}_{1} \mathrm{Cu}_{2} \mathrm{O}_{8+\delta}, \mathrm{Bi}(\mathrm{Pb})-2212$, with the maximum $T_{c} \simeq 93 \mathrm{~K}$, and the pure $\mathrm{Bi}-2212$ with the maximum $T_{c} \simeq 86 \mathrm{~K}$. Small mesa structures were made on freshly 

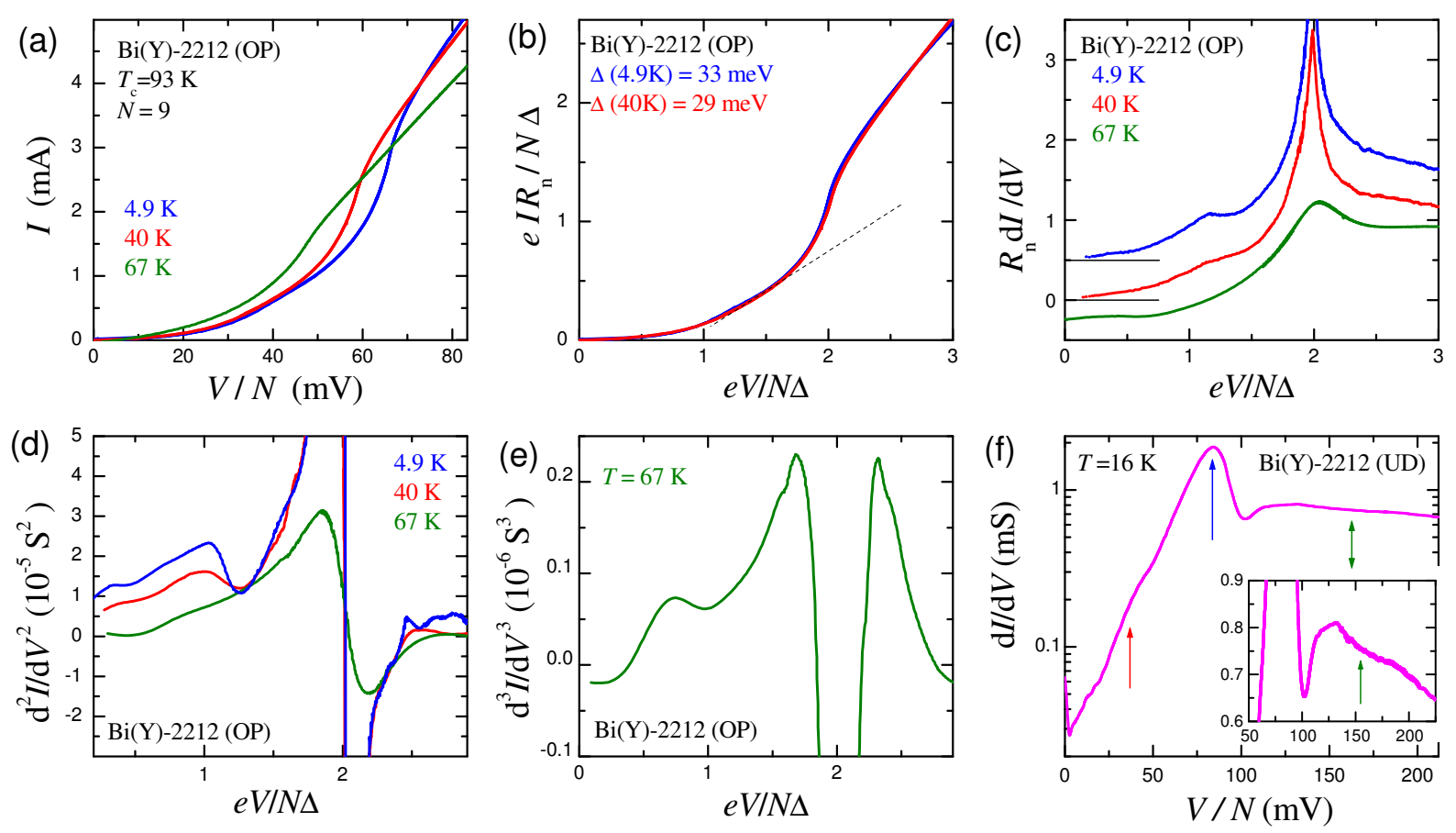

FIG. 1: (Color online). (a) Current-Voltage characteristics of an optimally doped (OP) $\operatorname{Bi}(\mathrm{Y})-2212$ mesa at different $T<T_{c}$. Only the last quasiparticle branches with all junctions in the resistive state are shown. (b) The same data, after normalization by $\Delta(T)$. The collapse of curves into one indicate that both voltage and current scales are determined by the gap value. Dashed line indicate a rapid increase of the sub-gap current at $e V / N>\Delta$. (c) $d I / d V(V)$ and (d) $d^{2} I / d V^{2}$ curves for the same data (the curves are shifted vertically for clarity). (e) Third-derivative characteristics at $T \simeq 67 \mathrm{~K}$. (f) The $d I / d V$ characteristics of a small underdoped (UD) $\mathrm{Bi}(\mathrm{Y})-2212$ mesa. Arrows indicate the subharmonic, sum-gap and four-gap singularities.

cleaved crystals using micro/nano-fabrication techniques. Details of mesa fabrication and characterization are described elsewhere 23 26. All presented measurements are performed at ambient magnetic field. The doping state of mesas was determined from a systematic study of all the characteristics, including (but not only) the $T_{c}$. Details, including the raw experimental data for different doping states can be found in Refs. [13, 14, 46]. In particular, in Ref. 14 oxygen-doped mesas from the same batch were studied. It was shown that the critical current of the mesas strongly (almost exponentially) depends on doping. This provides an accurate way of determination of doping close to optimal doping where $T_{c}$ vs. doping is flat.

Figure 1 (a) shows the current-voltage $(I-V)$ characteristics of a near optimally doped $\mathrm{Bi}(\mathrm{Y})-2212$ mesa with $T_{c} \simeq 93 \mathrm{~K}$ and $N=9$ intrinsic Josephson junctions, at $T=4.9,40$ and $67 \mathrm{~K}$. A pronounced current step occurs at the sum-gap voltage $V_{s g} / N=2 \Delta / e$, followed by the ohmic and almost $T$-independent resistance 24]. Such a behavior is typical for superconducting tunnel junctions 25]. Fig. 1 (b) demonstrates that the $I-V$ curves at different $T$ merge in one when both the voltage and the current scales are normalized by $\Delta(T)$. This is expected for SIS junctions, in which not only the voltage, but also the current scale is proportional to the superconducting gap, as seen from theoretical $I-V$ curves in Fig. 3 (a).
Fig. 1 (c) shows normalized $d I / d V(V)$ characteristics for the same mesa. A sharp sum-gap peak occurs at $e V_{s g} / N \Delta=2$. Simultaneously, we notice an additional bump at a half of the peak voltage, $e V / N \Delta \simeq 1$. This subharmonic gap feature will be in focus of this work. The subharmonic feature is rapidly smeared out with increasing temperature, but can be traced using higher derivatives $d^{2} I / d V^{2}$, Fig. 11(d), and $d^{3} I / d V^{3}$, Fig. 1](e).

With decreasing doping the sum-gap peak is decreasing in amplitude [13, 14. Fig. 1](f) shows a $d I / d V$ characteristics of a moderately underdoped $\mathrm{Bi}(\mathrm{Y})-2212$ mesa with $T_{c} \simeq 91 \mathrm{~K}$ at $T=16 \mathrm{~K}$ (note the semi-logarithmic scale). It is seen that for the underdoped mesa both the sum-gap peak and the subharmonic bump have significantly smaller amplitudes than for the near optimally doped case, Fig. 1 (c).

The sum-gap and the subharmonic singularities are not the only spectroscopic features in intrinsic tunneling characteristics. A double-arrow in Fig. 1 (f) points at a small dip in conductance, which occurs at approximately twice the sum-gap peak voltage, i.e., $e V / N \sim 4 \Delta$. The inset in Fig. 1 (f) shows a zoom-in on this feature. As discussed in Refs. [30, 31, this dip is caused by reabsorbtion of nonequilibrium bosons generated upon relaxation of injected electrons.

Figure 2 demonstrates doping dependence of the subharmonic singularity. Fig. 2 (a) shows sub-gap parts 

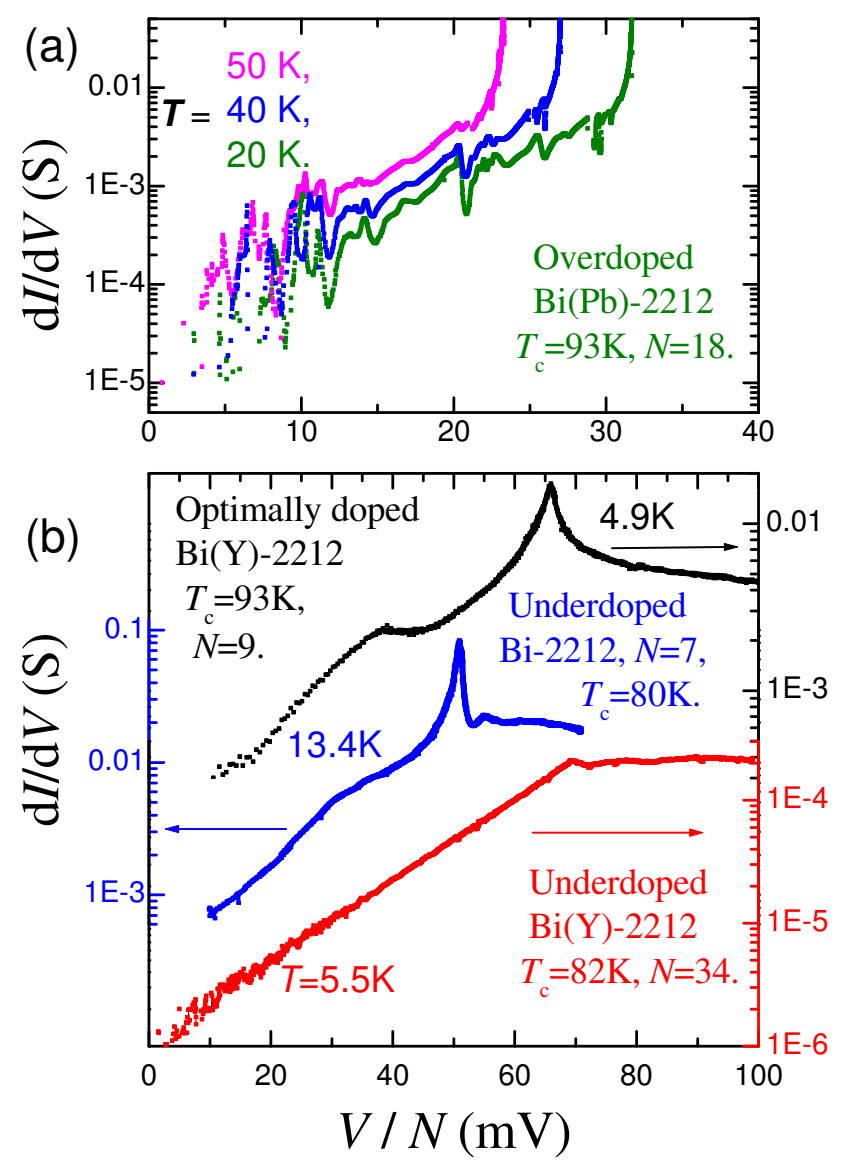

FIG. 2: (Color online). $d I / d V$ characteristics versus voltage per junction for mesas with different doping levels. (a) Sub-gap parts of $d I / d V$ for an overdoped $\mathrm{Bi}(\mathrm{Pb})-2212$ mesa. Strong singularities are due to phonon resonances. Note that positions of phonon resonances are temperature independent. (b) $d I / d V$ curves for near optimally doped $\mathrm{Bi}(\mathrm{Y})-2212$ (black), slightly underdoped Bi-2212 (blue) and moderately underdoped $\mathrm{Bi}(\mathrm{Y})-2212$ (red) mesas. Note that the subharmonic singularity disappears with decreasing doping.

of differential conductance $d I / d V$ vs voltage per junction for an overdoped $\mathrm{Bi}(\mathrm{Pb})-2212$ mesa. It is seen that in overdoped crystals the sub-gap conductance is dominated by strong phonon resonances [27 29], which appear at temperature-independent voltages. Presence of phonon resonances makes it difficult to analyze the subharmonic gap structure. Fig. 2 (b) shows $d I / d V(V / N)$ curves for optimally doped and underdoped mesas. It is seen that with decrease of doping the subharmonic feature is strongly decreased and gets completely washed away in moderately underdoped crystals [23, 24, while the sum-gap peak is still clearly visible. This may indicate changes in interlayer transport mechanism with doping [23].

A pronounced subharmonic gap structure at $V_{n}=$ $2 \Delta / e(n+1), n=1,2, \ldots$ has been observed in superconducting point contacts 32 and SIS junctions with pinholes 20]. The subharmonic structure is usually attributed to multiple Andreev reflections in quasi onedimensional quantum channels 21. The $d$-wave symmetry of the order parameter in cuprates should not destroy the subharmonic structure, but can affect its shape. In particular, it can cause an asymmetry between odd and even $n$ singularities as well as certain smearing due to angular dependence of the gap 33. The sub-gap structure has indeed been observed in cuprate junctions 34 37, although not all of it could be ascribed to Andreev reflections. For Bi-2212 cuprates the subharmonic gap structure has not been observed sofar neither in point contacts [38, nor in intrinsic junctions, although some excess subgap noise was reported for the latter [39], which might be related to Andreev reflections 33 .

A single sub-gap feature reported here is hard to reconcile with one-dimensional pinholes in the barrier, for which one would expect to see a series of subharmonic singularities [20. Rather it closely resembles the characteristics of homogeneous two-dimensional SINIS junctions [16 18. In SINIS junctions quasiparticles can travel at arbitrary angles with respect to the interface. The corresponding angular averaging leads to a significant smearing of the subharmonic structure so that only a leading $n=1$ singularity remains distinguishable. Thus, observation of a single subharmonic feature may indicate presence of a finite metallic conductivity in $\mathrm{BiO}$ layers.

This, however, is not the only possible interpretation. A similar single subharmonic feature at $\mathrm{eV}=\Delta$ occurs also in SIS junction when $\mathrm{S}$ is a gapless superconductor with a finite electronic density of states at the Fermi level. The gaplessness can originate from nodes in the gap in combination with partly incoherent (momentum non-conserving) tunneling and from impurity scattering. Fig. 3 presents numerical simulations for a gapless SIS junction, in which the finite density of states at the Fermi level was introducing adding a depairing factor (inverse quasiparticle lifetime) $\Gamma=2 \mathrm{meV}$ to the conventional BCS density of states (for details see e.g., the Supplementary material to Ref. [23]). Fig. 3 (a) shows calculated $I$ - $V$ curves at $T=4.9 K$ and $40 K$, with both $I$ - and $V$ scales normalized by $\Delta(T)$. Gaps and temperatures are the same as in Fig. 1 (a) to facilitate a direct comparison. A single subharmonic singularity can be seen as an approximately linear upturn of the quasiparticle current at $V \gtrsim \Delta / e$, indicated by dashed lines in Figs. 1 (b) and 3 (a). The corresponding $d I / d V(V), d^{2} I / d V^{2}$ and $d I^{3} d V^{3}$ curves, normalized by $\Delta(T)$, are shown in Figs. 3 (b), (c) and (d), respectively. Those theoretical curves are consistent with experimental data from Figs. 1 (c-e). The subharmonic feature in this case is entirely due to gaplessness and has the same origin as the singularity at $e V=\Delta$ in SIN junctions.

Thus, a single half-gap singularity is expected both in SINIS junctions with finite density of states in the intermediate N-layer and in gapless SIS junctions with finite density of states at the Fermi level in the S-layers. For our intrinsic junctions those two cases would correspond 

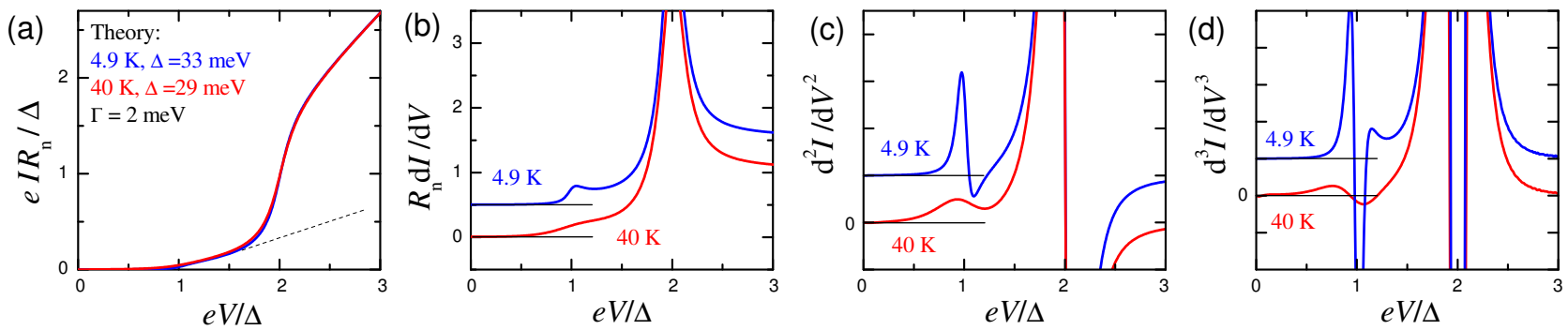

FIG. 3: (Color online). Numerically calculated characteristics for a gapless SIS junction with a finite depairing factor $\Gamma=2$ meV and with parameters of optimally doped $\mathrm{Bi}(\mathrm{Y})-2212$ mesa from Fig. 1 . (a) $I-V$ characteristics at $T=4.9$ and $40 \mathrm{~K}$ scaled by $\Delta(T)$. (b), (c) and (d) first, second and third derivatives (curves for different $T$ are shifted vertically for clarity). Note appearance of the sub-harmonic singularity at $e V=\Delta$ due to the gaplessness.
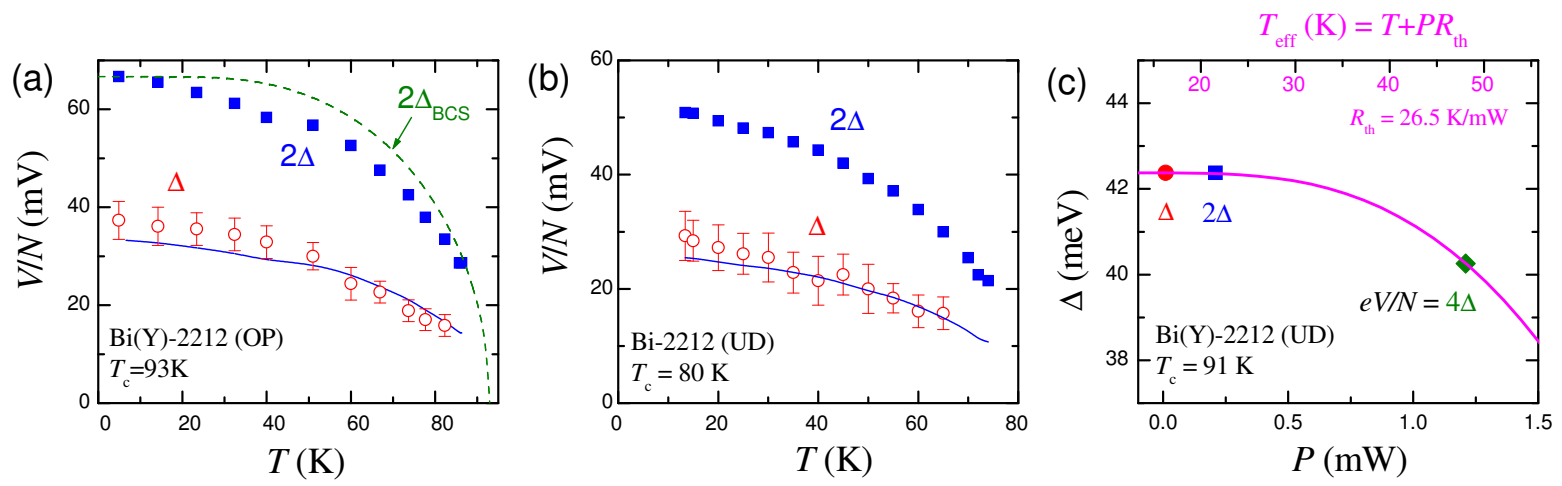

FIG. 4: (Color online). (a) and (b) Temperature dependencies of the sum-gap (squares) and the sub-gap singularities for (a) an optimally doped $\mathrm{Bi}(\mathrm{Y})-2212$ mesa from Fig. 1, and (b) for an underdoped Bi-2212 mesa. Solid lines represents half the sum-gap voltage. The dashed line in (a) shows the conventional BCS T-dependence of the superconducting gap. (c) Dissipation power dependence of the gap extracted from the sub-harmonic (circle) sum-gap (square) and four-gap (rhombus) singularities for a small underdoped $\mathrm{Bi}(\mathrm{Y})-2212$ mesa from Fig. 1 (f). Solid line represents the BCS gap at the effective mesa temperature.

to a finite metallic density of states in $\mathrm{BiO}$ layers or to a gapless case with a finite density of states in $\mathrm{CuO}_{2}$ layers. It is difficult to discriminate those scenarios just by looking at the shapes of $d I / d V(V)$ curves because the latter look very similar in both cases. However, a certain discrimination between those two scenarios can be made from the analysis of doping evolution of the singularity. As seen from Fig 2 (b), the subharmonic singularity is rapidly decreasing with decreasing doping. From angular-resolved photoemission [40, 41] and surface tunneling spectroscopy 42] it is known that the depairing factor $\Gamma$ is increasing with decreasing doping. Therefore, for the gapless SIS junction scenario the residual density of states at the Fermi level should not decrease with decreasing doping and the subharmonic singularity should still remain visible in underdoped junctions, which is not the case. To the contrary, for the SINIS scenario with $\mathrm{BiO}$ being the N-layer, it is expected that the density of states in the $\mathrm{BiO}$ layer will gradually decrease with decreasing doping and will eventually vanish upon approaching the insulating state. Consequently, the observed disappearance of the subharmonic singularity in moderately underdoped intrinsic junctions is consistent with SINIS case and implies presence of finite metallic properties in $\mathrm{BiO}$ layers.

Figures 4 (a) and (b) show $T$ - dependencies of the sum-gap voltage (solid squares) and the sub-gap singularity (open circles) for (a) the optimally doped $\mathrm{Bi}(\mathrm{Y})$ 2212 mesa from Fig. 1 and (b) for a moderately underdoped Bi-2212 mesa with $T_{c} \simeq 80 K$, studied in Ref. 23. The solid lines represent half of the sum-gap voltage and demonstrates that the sub-gap feature indeed represents the subharmonic half-gap singularity, rather than phonon resonances, which are $T$-independent [28, 29], as seen from Fig. 2 (a).

Observation of the subharmonic singularity allows accurate extraction of the genuine gap value, not affected by self-heating. Indeed, due to a smallness of the subgap current, the subharmonic singularity corresponds to a very small dissipation power and, therefore, is free from self-heating. For example, for the small $\mathrm{Bi}(\mathrm{Y})-2212$ mesa from Fig. 1 (f), $P=0.01 \mathrm{~mW}$ at the sub-gap, $0.21 \mathrm{~mW}$ at the sum-gap and $1.21 \mathrm{~mW}$ at the four gap singularities. Fig. 4 (c) shows gap values obtained from those three singularities as a function of the dissipation power. Due to self-heating [24, 43] the gap is gradually decreas- 
ing with increasing $P$ because the effective temperature of the mesa $T_{\text {eff }}=T+R_{t h} P$ is elevated above the base temperature $T$. Here $R_{t h}$ is the thermal resistance of the mesa [24, 43. The solid line in Fig. 4(c) represents the BCS $\Delta$ vs $T_{\text {eff }}$ dependence (top axis) obtained using $R_{t h}$ as a fitting parameter. The corresponding $R_{t h}=26.5$ $\mathrm{K} / \mathrm{mW}$ is in good agreement with the values obtained by in-situ measurements of self-heating [43] and from the analysis of size-dependence of intrinsic tunneling characteristics 24] on similar mesas.

From Fig. 4 (c) it is seen that the energy gap can be confidently obtained from intrinsic tunneling spectroscopy on small Bi-2212 mesas within more than two orders of magnitudes of the dissipation power. In all cases, reported here, self-heating at the subharmonic singularity is negligible. Therefore, this singularity provides a decisive self-consistency test and confirms accurate extraction of the energy gap by intrinsic tunneling spectroscopy made on small mesas [24].

To conclude, we reported on observation of subharmonic half-gap singularity in interlayer tunneling characteristics of Bi-2212 cuprates. The subharmonic singularity allows unambiguous determination of the energy gap because it occurs at a very small sub-gap current and negligible self-heating. This is an important step for development of intrinsic tunneling spectroscopy of cuprates. We have argued that the subharmonic singularity is a manifestation of the finite electronic density of states at the Fermi level in the superconducting state. The observed doping dependence indicated that the phenomenon is most likely brought forward by metallic behavior of intermediate $\mathrm{BiO}$ layer. The latter may have a significant influence on properties of layered cuprates. For example, it is well established that the anisotropy in cuprates is strongly doping dependent [1]. For Bi-2212 it changes from about a million in underdoped to a hundred in overdoped state. For $\mathrm{YBa}_{2} \mathrm{Cu}_{3} \mathrm{O}_{7-x}$ it changes from about a hundred in underdoped (which even exhibit the intrinsic Josephson effect [4]) to about five in a slightly overdoped case. Such a behavior can be explained by a gradual enhancement of metallic properties of intermediate layers with increasing doping, which adds a coherent mechanism to interlayer transport, and provides a way for establishing a fully coherent three-dimensional $c$-axis transport in the strongly overdoped metallic (Fermi liquid) state. To the contrary, in the underdoped state the metallic behavior of $\mathrm{BiO}$ layers becomes weak. When the sheet resistance exceeds the quantum resistance, $h / e^{2}$, a metal-insulator transition takes place due to Coulomb blocking of transport 44. The corresponding Coulomb energy can represent one of the contribution to the $c$-axis pseudogap phenomenon and is consistent with recent observation of an additional "dressed" electron energy in interlayer tunneling characteristics of underdoped Bi-2212 46.

\section{Acknowledgments}

The work was supported by the Swedish Foundation for International Cooperation in Research and Higher Education Grant No. IG2013-5453 and the Swedish Research Council Grant No. 621-2014-4314. I am are grateful to S.O. Katterwe for assistance in experiment.
[1] T. Watanabe, T. Fujii, and A. Matsuda, Phys. Rev. Lett. 79, 2113 (1997).

[2] P.W. Anderson and Z.Zou, Phys. Rev. Lett. 60, 132 (1988).

[3] R.H. McKenzie and P.Moses, Phys. Rev. Lett. 81, 4492 (1998).

[4] M. Turlakov and A.J. Leggett, Phys. Rev. B 63, 064518 (2001).

[5] W. Kim and J.P. Carbotte, Phys. Rev. B 63, 054526 (2001).

[6] M. Ferrero, O. Parcollet, A. Georges, G. Kotliar, and D. N. Basov, Phys. Rev. B 82, 054502 (2010).

[7] S.A. Hartnoll, Nature Phys. 11, 54 (2015)

[8] R. Kleiner and P. Müller, Phys. Rev. B 49, 1327 (1994).

[9] A. A. Kordyuk, S. V. Borisenko, T. K. Kim, K. A. Nenkov, M. Knupfer, J. Fink, M. S. Golden, H. Berger, and R.Follath, Phys. Rev. Lett. 89, 077003 (2002).

[10] B. Vignolle, B.J. Ramshaw, J. Day, D. LeBoeuf, S. Lepault, R. Liang, W.N. Hardy, D.A. Bonn, L. Taillefer, and C. Proust, Phys. Rev. B 85, 224524 (2012).

[11] A. Glatz, A. A. Varlamov, and V. M. Vinokur, Phys. Rev. B 84, 104510 (2011).

[12] Y. Tanaka and S. Kashiwaya, Phys. Rev. B 56, 892 (1997).
[13] V.M.Krasnov, Phys. Rev. B 65, 140504(R) (2002).

[14] V.M. Krasnov, Phys. Rev. B 91, 224508 (2015).

[15] S. O. Katterwe, A.Rydh, H. Motzkau, A.B. Kulakov, and V. M. Krasnov Phys. Rev. B 82, 024517 (2010).

[16] A.Brinkman, and A.A. Golubov, Phys. Rev. B 61, 11297 (2000).

[17] D. Cassel, G. Pickartz, M. Siegel, E. Goldobin, H.H. Kohlstedt, A. Brinkman, A.A. Golubov, M. Yu. Kupriyanov, and H. Rogalla, Physica C 350, 276-290 (2001).

[18] S. Rajauria, P. Gandit, T. Fournier, F.W. J. Hekking, B. Pannetier, and H. Courtois, Phys. Rev. Lett. 100, 207002 (2008).

[19] M. Octavio, M. Tinkham, G. E. Blonder, and T. M. Klapwijk, Phys. Rev. B 27, 6739 (1983)

[20] A.W. Kleinsasser, R.E. Miller, W.H. Mallison, and G.B. Arnold, Phys. Rev. Lett. 72, 1738 (1994).

[21] T. Lofwander, V.S. Shumeiko, and G. Wendin, Supercond. Sci. Technol. 14, R53R77 (2001).

[22] A. F. Andreev, Sov. Phys. JETP 19, 1228 (1964)

[23] S.O. Katterwe, A.Rydh, and V. M. Krasnov, Phys. Rev. Lett. 101, 087003 (2008).

[24] V. M. Krasnov, Phys. Rev. B 79, 214510 (2009).

[25] V. M. Krasnov, H. Motzkau, T. Golod, A. Rydh, S. O. 
Katterwe, and A. B. Kulakov, Phys. Rev. B 84, 054516 (2011).

[26] Th. Jacobs et.al., Phys. Rev. B 86, 214506 (2012).

[27] K.Schlenga et al., Phys. Rev. B 57, 14518 (1998)

[28] Ya.G. Ponomarev et al., Solid State Commun. 111, 513 (1999).

[29] S.O. Katterwe, H. Motzkau, A. Rydh, and V.M. Krasnov, Phys. Rev. B 83, 100510(R) (2011).

[30] V.M. Krasnov, Phys. Rev. Lett. 97, 257003 (2006).

[31] V. M. Krasnov, S.O. Katterwe1, and A. Rydh, Nature Commun. 4, 2970 (2013); DOI: 10.1038/ncomms3970.

[32] E. Scheer, J.C. Cuevas, A. Levy Yeyati, A. MartinRodero, P. Joyez, M.H. Devoret, D. Esteve, C. Urbina, Physica B 280, 425 (2000).

[33] A. Poenicke, J. C. Cuevas, and M. Fogelström, Phys. Rev. $B$ 65, 220510(R) (2002).

[34] G. Deutscher, Nature 397, 410 (1999).

[35] G. Koren and T. Kirzhner, Phys. Rev. B 84, 134517 (2011).

[36] J.H. Ngai, W.A. Atkinson, and J.Y.T. Wei, Phys. Rev. Lett. 98, 177003 (2007).

[37] T. Imaizumi, T. Kawai, T. Uchiyama, and I. Iguchi, J. Low Temp. Phys. 131, 809 (2003).
[38] L. Ozyuzer, J.F. Zasadzinski, C. Kendziora, and K.E. Gray, Phys. Rev. B 61, 3629 (2000).

[39] A. Saito, H. Ishida and K. Hamasaki, A. Irie and G. Oya, Appl. Phys. Lett. 85, 1196 (2004).

[40] M. Norman, M. Randeira, H. Ding, and J. C. Campuzano, Phys. Rev. B 57, R11093 (1998).

[41] I. M. Vishik, E. A. Nowadnick, W. S. Lee, Z. X. Shen, B. Moritz, T. P. Devereaux, K. Tanaka, T. Sasagawa and T. Fujii, Nature Phys. 5, 718 (2009).

[42] J.W. Alldredge et al., J. Lee, K. McElroy, M. Wang, K. Fujita, Y. Kohsaka, C. Taylor, H. Eisaki, S. Uchida, P.J. Hirschfeld and J.C. Davis, Nature Phys. 4, 319 (2008).

[43] V.M. Krasnov, M. Sandberg, and I. Zogaj, Phys. Rev. Lett. 94, 077003 (2005).

[44] B. Sacepe, C. Chapelier, T.I. Baturina, V.M. Vinokur, M.R. Baklanov, and M. Sanquer, Nature Commun. 1, 140 (2010).

[45] M. Nagao, S. Urayama, S. M. Kim, H. B. Wang, K. S. Yun, Y. Takano, T. Hatano, I. Iguchi, T. Yamashita, M. Tachiki, and H. Maeda, Phys. Rev. B. 74, 054502 (2006).

[46] Th. Jacobs, Y. Simsek, Y. Koval, P. Müller, and V. M. Krasnov, Phys. Rev. Lett. 116, 067001 (2016). 